\title{
Registration and immobilization in robot-assisted surgery
}

\author{
Jon T. Lea ${ }^{1}$,Dane Watkins ${ }^{1}$, Aaron Mills ${ }^{1}$, Michael A. Peshkin ${ }^{1}$, \\ Thomas C. Kienzle III $^{2}$, S. David Stulberg ${ }^{2}$ \\ ${ }^{1}$ Department of Mechanical Engineering \\ Northwestern University \\ Evanston, IL 60208 \\ (708) 491-7470 \\ (708) 491-3915 fax \\ jonlea@nwu.edu \\ peshkin@nwu.edu \\ ${ }^{2}$ Department of Orthopedic Surgery \\ Northwestern University Medical School \\ Chicago, IL 60611
}

Support for this research was provided by Northwestern University, Northwestern Memorial Hospital and NSF PYI Grant DMC-8857854 (MP).

Much of this report appears in the conference proceedings of the First International Symposium on Medical Robotics and Computer Assisted Surgery, Pittsburgh, PA, Sept. 1994.

\begin{abstract}
Robotic systems for computer assisted surgery involve both tools and techniques which are new to the surgical arena. Registration and immobilization in particular are key problems. Registration is the spatial alignment of the coordinate frames of the robot, an anatomic object (e.g., a bone), and the preoperative plan (a computer model). Immobilization is necessary to maintain that alignment. We discuss various approaches to registration and immobilization, and solutions appropriate for an orthopedic surgical system.
\end{abstract}

Keywords: Computer assisted surgery, Robotics, Registration, Immobilization, Fiducials

\section{INTRODUCTION}

With the aid of computer-based graphical surgical planning systems, robots are making their way into the operating room. Robotic systems are not intended as replacements for human doctors, but rather as smart surgical tools. Their most valuable function may be their capacity to create an information link from preoperative surgical plans to the surgical arena.

Most surgical robotic systems have distinct planning and surgical phases. During the planning phase, images from computed tomography (CT), magnetic resonance imaging (MRI), or conventional radiography ( 2D x-rays) are used to display a graphical model of the patient's anatomy. A surgical procedure is planned on this model and the numerical coordinates which describe that procedure are stored. During surgery, a robot physically displays the planned tool trajectory by positioning a template at the correct anatomic location on the patient, allowing the surgeon to execute the intervention with conventional surgical tools. Alternatively, the robot performs the intervention itself using a tool such as a pneumatic drill or burr.

Registration and immobilization in robot-assisted surgery

Jon T. Lea, Dane Watkins, Aaron Mills, Michael A. Peshkin, Thomas C. Kienzle III, S. David Stulberg Journal of Image Guided Surgery 1 (2), pp. 80-87, 1995 
Registration and immobilization are two central issues which must be addressed before a robot can position a template or tool accurately on a patient. Registration establishes the relative spatial relationships between the robot, the preoperative plan, and the involved anatomy of the patient. Immobilization maintains these relationships.

\section{Registration and Fiducials}

Registration is the determination of the spatial relationship of three coordinate frames $(x, y$, $\mathrm{Z}$ axes): that of the robot, that of an anatomic object in the robot's work space, and that of the preoperative plan (e.g., a plan made on a 3D reconstruction of CT data). When registration is complete, a designated position on the preoperative image can be translated into a set of robot joint angles. That set of joint angles will cause the robot to put a surgical tool at the position on the patient that corresponds to the designated position in the image. For a variety of practical reasons, registration procedures often have several steps, and involve registering some intermediate coordinate frames and artifacts in addition to the three essential ones listed above.

Registration graphs (introduced in Lea et al. ${ }^{8}$ ) are a diagrammatic technique for displaying and analyzing the procedures as well as the physical objects involved in registration in a computer assisted surgery system. The registration graph is a mathematical graph, that is, it consists simply of nodes and links. The purpose of registration graphs is to provide a connected path corresponding exactly to valid registration in the computer assisted surgery system under study. Whether or not a given pair of objects must be immobilized may also be determined from connectivity properties of the registration graph.

This paper does not address such "system" aspects of registration or immobilization, but rather concentrates on techniques and devices for individual steps in registration and immobilization. While much of the discussion which follows is in the context of our total knee replacement (TKR) system ${ }^{5,14}$, it is applicable to many orthopedic procedures.

\section{Artificial vs. anatomic fiducials}

A key element in registration is the use of fiducials. Fiducials are reference features, located on both the computer-based model and the anatomic object. The fiducials are used to relate the coordinate frames of the computer-based model and the anatomic object.

Most surgical robot systems to date use artificial fiducials, since they are more easily located in the preoperative images and by the robot. For instance, the neurosurgical system developed by Kwoh et al. ${ }^{6}$, uses a modified stereotactic headframe outfitted with "N"shaped fiducials. Cross-sections of the fiducials appear in each CT image and indicate the location of each image within the headframe. During surgery, the headframe is mounted at a known location with respect to the robot, providing a fixed, repeatable relationship between the patient and robot.

Several orthopedic systems, such as our total knee replacement system, a TKR system by Fadda et al. ${ }^{3}$, and the ROBODOC ${ }^{\mathrm{TM}}$ total hip replacement system described by Taylor et al. ${ }^{12}$, use titanium bone pins implanted in the femur as fiducials. The titanium pins are found in preoperative CT images and are used to identify a coordinate frame on the bone. The bone is immobilized during surgery and each pin is located physically with a probe attached to the robot end-effector.

Some studies are underway in the use of anatomic fiducials. In many instances, anatomic fiducials are preferable to artificial fiducials since they do not require an invasive implant 
procedure. Lavallée et al. ${ }^{7}$ have developed a surgical robot system for pedicle screw placement using an optical spatial localizer. The vertebra surface is the fiducial, and a probe is used to digitize points from the surface. These points are matched to a preoperative CT model. Laser ranging systems have been used by Grimson et al. ${ }^{4}$ and Péria et al. ${ }^{10}$ to digitize facial features (the fiducials) which are then matched to models from preoperative CT, MRI and SPECT (Single Photon Emission Computed Tomography) data. Fadda et al. ${ }^{2}$ have investigated a similar technique with a surgical robot TKR system. A laser ranging system mounted on an ancillary robot is used to scan the contour of the exposed bone. The resulting fiducial surface is matched to a preoperative CT model.

Simon et al. ${ }^{11}$ have developed techniques for fast anatomic registration, enabling registration (with devices such as laser ranging systems) to be done on unfixed anatomies at update rates up to $10 \mathrm{~Hz}$. The selection of intraoperative data sets which minimize the required number of digitized data points, without compromising accuracy, is also being investigated.

\section{Point and spatial fiducials}

Fiducials can also be categorized by their geometry, which determines the number of degrees of freedom that the fiducial identifies. Point fiducials and spatial fiducials are the most common, although linear and planar fiducials are theoretically possible.

A single point fiducial provides only three of the six constraints needed to define a coordinate frame (specifically, it provides an $\mathrm{x}, \mathrm{y}$, and $\mathrm{z}$ coordinate.) When using only point fiducials for registration, a minimum set of three fiducials is required to fully define a coordinate frame. As an example, our TKR system uses a combination of artificial and anatomic point fiducials. These fiducials are defined by four internally placed pins at the knee, an externally placed pin at the ankle, and an inferred point (see below) at the center of the femoral head (Figure 1). Together these six point fiducials define two coordinate frames, one for the femur and one for the tibia.

Spatial fiducials have geometries which provide all six spatial constraints needed to define a coordinate frame. Typically, a highly contoured surface is used. For example, the complex surface of the vertebra including the spinous and transverse processes is used as a spatial fiducial in the pedicle screw placement system of Lavallée et al. ${ }^{7}$. The three dimensional bone contour used in the system of Fadda et al. ${ }^{2}$ is another example of a spatial fiducial. In selecting spatial fiducials, the geometry must be complex enough so to avoid degeneration. For example, although the spherical surface of the femoral head (used in our TKR system) is a spatial fiducial, it only provides three constraints (an $\mathrm{x}, \mathrm{y}, \mathrm{z}$ position), and thus degenerates to a point fiducial.

\section{Immobilization}

It is not enough to identify the fiducials and establish registration at a particular instant; registration must be maintained over the course of the surgery. There are two primary methods for maintaining registration: immobilization and tracking. Immobilization involves fixing the bones with respect to the robot. Alternatively, the positions of the bones could be tracked (either by the robot or a secondary device). The pedicle screw placement systems of Lavallée et al. ${ }^{7}$ and Nolte et al. ${ }^{9}$ employ this technique by placing an infrared LED array on the spinous process and tracking vertebra motion with a spatial localizer. However, many orthopedic procedures, especially those involvong the larger bones of the 
skeletal system, are suited to immobilization since the bones are rigid structures which can be clamped.

\section{Accuracy requirements}

Immobilization at the surgical site is most critical to maintain the desired accuracies. Any relative motion between structures at the surgical site and the robot will directly translate into errors in fiducial registration or errors in the robotic positioning of a surgical tool. However, immobilization of the structures far from the surgical site need not be so rigorous, since the distant fiducials primarily provide directional information for the coordinate frames associated with the bones. In general, the further fiducials are from the surgical site, the less rigorous the required immobilization. For example, on an average length femur, a $3 \mathrm{~mm}$ displacement error of the femoral head will result in a $0.5^{\circ}$ orientation error at the knee joint.

\section{Partially constraining vs. fully constraining immobilization}

Fully constraining immobilization has been used in most surgical robot systems. In the neurosurgical robot system described in Kwoh et al. ${ }^{6}$, the patient's skull is rigidly attached to the stereotactic headframe, and the headframe is mounted rigidly to the base of the robot. Thus all motions of the head with respect to the robot are constrained. The ROBODOC ${ }^{\mathrm{TM}}$ system $^{12}$ uses a large external bone clamp originating from the base of the robot. The clamp is attached to the shaft of the femur through the muscles. Fixing to external surfaces is appropriate in these cases where the intervention is internal to the immobilized bone.

Partially constraining immobilization is necessary to constrain certain motions of bones while permitting other motions. This type of immobilization is necessary in our TKR system during femur tracking. Inhibition of translations of the center of the femoral head are required, while allowing flexion/extension and abduction/adduction of the femur (i.e., pivoting about the center of the femoral head).

\section{MATERIALS AND METHODS}

\section{Computer Assisted Total Knee Replacement Surgery}

Total knee replacement surgery (TKR) involves replacing the articular surfaces of the knee joint (both femur and tibia) with titanium and polyethylene components. The external face of each prosthetic component is similar in shape to the knee surface it replaces. When implanted correctly, the components restore the complex hinge-like motion of the knee. The components are positioned in the knee by seating them correctly on resected planes on each bone.

To improve the surgical outcome of these procedures, we have developed an integrated system consisting of a graphics workstation for preoperative planning and a computercontrolled robot for assistance during surgery ${ }^{5,14}$. Preoperatively, CT-based threedimensional (3D) models of the patient's femur and tibia are used to plan the placement of the prosthetic components. The required bone resections are displayed on screen and can be modified according to the surgeon's clinical judgment. In the operating room, a robot positions a cutting/drilling guide against the bone at the location and orientation of each resection, allowing the surgeon to perform the required resections with a conventional bone saw. 
In developing this surgical robot system, we have addressed the issues of registration and immobilization. This has included development of registration artifacts (fiducial pins), a suitable method for registration between the patient, the robot, and the CT-based preoperative plan, and specialized devices for holding the involved bones while the robot performs some specific actions.

\section{TKR Artificial Point Fiducials}

For TKR surgery, internally placed fiducial pins in the knee provide accuracy at the surgical site since they can be located precisely. During a preoperative visit, the surgeon percutaneously implants two small fiducial pins in the distal femur. Two small fiducial pins are placed in the proximal tibia in a similar manner.

There are many requirements on the design of the fiducial pins. The pins must firmly hold the thin cortical bone and the underlying soft cancellous bone, yet be small enough to be inserted through the skin as an office procedure. The pins should mount flush with the bone so the head of the pin does not irritate nearby tissues. They should be easily accessed and located with the robot. Further, the pins should not introduce significant distortions in the CT images.

Our fiducial pins are modified titanium screws (which maximize clarity in the CT image) with a chisel point in the tip and a recessed cone in the head. A standard hex-head drive tool allows the surgeon to insert the pins into the patient's bone. The recessed cone provides a mating surface for the robot mounted probe during registration in the operating room and defines the exact point of the fiducial (Figure 2).

The use of an external artificial fiducial at the distal end of the tibia makes an invasive procedure there unnecessary. During the preoperative office visit, a fiberglass cast is fit around the patient's ankle. Once hardened, the cast is carefully removed and a fiducial pin is placed in the cast over the medial malleolus.

\section{An Inferred Anatomic Point Fiducial}

Assigning a third fiducial to the proximal femur presents a challenge. The thigh is surrounded by soft tissue (muscle and adipose) which prevents any artificial fiducial on the skin surface from being consistently and reliably located with respect to the bone. Further, when the fiducials are far from the surgical site, invasive techniques for implantation or registration are undesirable. Therefore the third fiducial chosen is the center of the head of the femur. As noted earlier, this is actually a spatial fiducial (the femoral head surface) which degenerates to a point fiducial because of its symmetry. This point fiducial is found in the CT-based model by identifying several points on the spherical face of the femoral head and calculating its center.

During surgery, the robot must also find the location of this anatomic point fiducial. With the robot clamped to the knee (using the intramedullary bone clamp described below), the surgeon manually moves the entire leg (which is able to pivot only about the femoral head) through substantial arcs, while the attached robot samples positions. This dynamic registration process, known as femur-tracking, allows the center of the femoral head to be inferred as the center of a sphere fit to the recorded positions of the robot endpoint.

Embedding internal artificial fiducials at the knee is appropriate for TKR, since the surgery directly involves the knee. By using an inferred anatomic fiducial at the center of the femoral head and using an external artificial fiducial at the ankle, we avoid invasive procedures at areas not directly involved in the surgery. 
The method by which a coordinate frame is assigned to the fiducial set can affect the accuracy of the registration. Figure 3 shows the coordinate frame for the femur in our TKR system. The three point fiducials provide nine constraints on the femur. However, only six constraints are needed to determine the location and orientation of the femur. We resolve this redundancy in such a way that errors in the center of the femoral head (which we expect to be several millimeters) contribute as little as possible to the six variables of interest.

First, the two fiducial pins in the distal femur define a medial-lateral axis. The proximaldistal axis is defined to lie in the plane of the triangle formed by all three fiducial pins, and to be perpendicular to the medial-lateral axis. The anterior-posterior axis is simply perpendicular to the other two axes. Finally, the origin of the coordinate frame is located midway between the fiducial pins of the distal femur.

\section{Surgical Accuracies and Fixturing Devices}

The accuracies required for any particular procedure are specific to the surgery. Thus, the demands on registration and immobilization (or tracking) are dependent on the type of surgery. This results in different fixing devices and techniques for each surgery.

In TKR, the current accuracy of prosthetic placement using conventional (non-robotic) jigbased systems is within $2-3^{\circ}$ of the intended varus/valgus orientation ${ }^{1}$ (also, Stulberg personal communication). The accuracy of other rotational and translational placements (most importantly, proximal/distal placement and internal/ external rotation) is less well known, since they are not planned preoperatively but are determined by the particular design of the jig system being used and by the surgeon's personal technique.

Control over all rotational and translational placements is desirable, since optimal placement will enhance the postoperative function of the knee joint. During preoperative planning, optimal placement of the prosthetic components based on topographic (bone surface), geometric (anatomic bone relationships), or dynamic (leg mobility) requirements is possible. With our robot-assisted system, the goal is accuracies within $1^{\circ}$ and $1 \mathrm{~mm}$ of all intended rotational and translational placements, respectively.

These requirements led us to the development of three specialized fixing devices for immobilization of the bones. At the knee joint (for both the femur and tibia) a long rod (the intramedullary bone clamp) is driven into the medullary canal, and a rigid fixator arm mounted next to the robot holds this rod fixed. The proximal end of the femur is immobilized by holding the pelvis with a "hipband" structure attached to the surgical table (Figure 4). The distal end of the tibia is immobilized using a modified Mark II Stulberg leg positioner which can be directly attached to the surgical table (Figure 5).

The intramedullary bone clamp consists of a stainless steel rod $40 \mathrm{~cm}$ in length and $9.5 \mathrm{~mm}$ in diameter, and a tapered stainless steel cone which slides along the rod. Once the rod is driven into the medullary canal, the cone is wedged into the bone and locked to the rod. The fixator arm is connected to the protruding end of the rod and then locked down. This provides very rigid immobilization at the knee joint. The device allows easy access with the robot to the fiducial pins and clear display of the resection angles.

In immobilizing the pelvis, full rotational range of motion of the hip joint is allowed to flex the leg during femur-tracking, but translational motion of the pelvis is prevented. A vacuum pack, a commercially available bag that hardens and molds to a contour when air is removed, is used under the lower back of the patient during surgery to prevent pressure 
sores, without sacrificing rigidity. Downward pressure is applied to the pelvis at three areas (along each anterior superior iliac spine and the pubic symphysis) using anatomically contoured, foam covered aluminum blocks attached to an adjustable pressure frame which connects to the surgical table. The contours of the aluminum blocks are designed to provide maximum clamping force while avoiding application of high pressure points to the skin and resultant tissue necrosis ${ }^{13}$.

Since the distal tibia has an externally located fiducial pin (in the fiberglass cast), dynamic fiducial location is not necessary and the fixing can fully constrain the leg. The ankle is tightly wrapped to the foot/ankle support of a modified Mark II Stulberg leg positioner which is clamped to the surgical table. The fiducial pin is left exposed and is located in the same manner as the fiducial pins at the knee.

\section{RESULTS}

\section{Intramedullary Bone Clamp and Fixator Arm}

The intramedullary bone clamp has been tested on several femur and tibia specimens. The force required to pull the rod out of the bone was in excess of $30 \mathrm{lbf}$ on the most conservative trial. The clamping rod has also been tested on a human patient, and was accepted by the surgeon as a practical tool for this application.

The fixator arm provides very rigid immobilization at the knee joint. The endpoint stiffness has been determined by applying a $5 \mathrm{lbf}$ load (which is an estimate of the force a surgeon may apply during surgery) normal to the axis of the arm at its maximum extension (24"). The arm deflects only 0.03 " under the $5 \mathrm{lbf}$ load $(30 \mathrm{~N} / \mathrm{mm})$. Additionally, displacements caused be tool forces (e.g., a drill bit or powered sagittal saw) occur in directions which do not significantly affect the positioning of robot held template. Properly used, the fixator arm is sufficiently stiff to maintain immobilization at the knee.

\section{Hip Immobilizer}

Because of the unique requirements of the pelvic fixing device (hipband), a series of hip immobilization tests were undertaken on several cadaveric specimens. Three threaded rods ( $25 \mathrm{~cm}$ long, $10 \mathrm{~mm}$ in diameter) were inserted into the pelvis: one each through the left and right ilium extending laterally and the third through the right pubic ramus extending anteriorly. After immobilizing the pelvis using the hipband and vacuum pack, rod endpoint displacements were measured using dial indicators while the femur was moved. Femur motions started from a zero position $\left(0^{\circ}\right.$ abduction and $0^{\circ}$ flexion) to a given adduction/ abduction angle, and then to several flexion angles.

The rod endpoint displacements were used to compute translations of the center of the femoral head. By studying these motions with respect to a femur fixed coordinate frame (with the origin at the center of the femoral head), we found that the proximal/distal displacements can be neglected (since they will not affect the resultant orientation accuracy at the knee joint). The medial/lateral and anterior/superior displacements (which are orthogonal to the long axis of the femur) can be shown on a vector plot (Figure 6). Note that this coordinate frame is not the same as the registration coordinate frame shown in Figure 3. While the vector plots show general trends in the displacement directions, they are not repeatable since it is difficult to immobilize the pelvis in the exact same manner from test to test. However, the magnitudes are somewhat repeatable and the largest displacements were approximately $5 \mathrm{~mm}$ on all tests. The vector plots suggest preferred angles of motion for minimizing displacement errors during femur tracking and show that 
the femoral head can be localized within 2-3 mm. Finally, the displacements of the femoral head in an actual patient should be substantially lower since cadavers are relatively inflexible.

\section{Ankle Immobilizer}

The primary source of error in locating the external fiducial pin at the ankle comes from the remounting the fiberglass ankle cast. Since the ankle immobilizer is a fully constraining device, accuracy is not significantly compromised. The ankle immobilizer has performed well in our tests.

\section{CONCLUSION}

By discussing the registration techniques and immobilization devices for our total knee replacement system in detail, we have illustrated many of the design criteria that must be considered in computer assisted surgery. Also, the discussion demonstrates the effect required accuracies have on registration and immobilization. In developing systems for new robot-assisted procedures, it is important to consider these factors.

Registration techniques using artificial fiducials typically yield higher accuracies than techniques employing anatomic fiducials, since artificial fiducials are highly defined in preoperative diagnostic images and can be exactly located by the robot. However, as matching algorithms and techniques for anatomic fiducial registration are further refined, non-invasive methods of registration hold much promise. Artificial and anatomic fiducials can be used together to achieve high accuracies while avoiding invasive procedures at uninvolved sites on the patient.

Immobilization requirements are currently fairly strict (that is, the fixing devices must rigidly hold the anatomic structures). These requirements will be relaxed as real-time registration techniques, which utilize tracking devices, are developed. Real-time registration guarantees that if, for instance, a bone moves with respect to the robot after the initial registration was established, corrections can be made to the relative transformation between the robot and bone and thus the errors avoided. While it may seem that tracking devices will elimate the need for immobilization, errors introduced from tracking latencies are minimized through appropriate fixation.

\section{REFERENCES}

1. Engh GA, Peterson BE (1990) Comparative experience with intramedullary and extramedullary alignment in total knee arthroplasty. J Arthroplasty 5:1.

2. Fadda M, Martelli S, Dario P, Marcacci M, Visani A, Zaffagnini S (1992) First steps towards robot-assisted discectomy and arthroplasty. Innovation and Technology in Biology and Medicine 13:395-408.

3. Fadda M, Wang T, Marcacci S, Martelli S, Dario P, Marcenaro G, Nanetti M, Paggetti C, Visani A, Zaffagnini S (1994) Computer-assisted knee arthroplasty at Rizzoli Institutes. Proceedings of the First International Symposium on Medical Robotics and Computer Assisted Surgery 1:26-30.

4. Grimson WEL, Lozano-Pérez T, Wells WM, Ettinger GJ, White SJ, Kikinis R (1994) Automated registration for enhanced reality visualization in surgery. Proceedings of the First International Symposium on Medical Robotics and Computer Assisted Surgery 1:82-89. 
5. Kienzle TC, Stulberg SD, Peshkin MA, Quaid A, Wu CH (1993) An integrated CAD-robotics system for total knee replacement surgery. Proceedings of the 1993 IEEE International Conference on Robotics and Automation 1:889-894.

6. Kwoh, YS, Hou J, Jonckeere EA, Hayati S (1988) A Robot with improved absolute positioning accuracy for CT guided stereotactic brain surgery. IEEE Transactions on Biomedical Engineering 35:153-160.

7. Lavallée S, Sautot P, Troccaz J, Cinquin P, Merloz P (1994) Computer assisted spine surgery: A technique for accurate transpedicular screw fixation using CT data and a 3D optical localizer. Proceedings of the First International Symposium on Medical Robotics and Computer Assisted Surgery 2:315-322.

8. Lea JT, Santos-Munné JJ, Peshkin MA (1995) Diagramming registration connectivity and structure. IEEE Engineering in Medicine and Biology (in press).

9. $\quad$ Nolte L-P, Zamarano LJ, Jiang Z, Wang Q, Langlotz F, Arm E, Visarius H (1994) A Novel Approach to Computer Assisted Spine Surgery. Proceedings of the First International Symposium on Medical Robotics and Computer Assisted Surgery 2:323-328.

10. Péria O, François-Joubert A, Lavallée S, Champleboux G, Cinquin P, Grand S (1994) Accurate registration of SPECT and MR brain images of patients suffering from epilepsy or tumor. Proceedings of the First International Symposium on Medical Robotics and Computer Assisted Surgery 1:58-62.

11. Simon DA, Hebert M, Kanade T (1994) Techniques for fast and accurate intrasurgical registration. Proceedings of the First International Symposium on Medical Robotics and Computer Assisted Surgery 1:90-97.

12. Taylor, RH, Mittelstadt BD, Paul HA, Hanson W, Kazanzides P, Zuhars JF, Williamson B, Musits BL, Glassman E, Bargar WL (1994) An image-directed robotic system for precise orthopaedic surgery. IEEE Transactions Robotics and Automation 10:261-275.

13. Woolsey RM, McGarry JD The cause, prevention, and treatment of pressure sores. Neurol Clin 9:797-805.

14. Wu CH, Stulberg SD, Papaiannou J, Huang HY, Hwang KS, Kienzle TC (1993) An integrated CT-imaging, CAD-based system for orthopedic surgery. Proceedings of the 1993 IEEE International Conference on Robotics and Automation 1:895-900.

\section{FIGURE LEGENDS}

Figure 1. Five fiducial pins and the center of the femoral head provide the three necessary point fiducials per bone.

Figure 2. Registration of fiducial pins using the robot mounted probe.

Figure 3. Computerized bone model and coordinate frame through three point fiducials.

Figure 4. Immobilization of the femur using the intramedullary bone clamp, fixator arm, and hip immobilizer. 
Figure 5. Immobilization of the tibia using the intramedullary bone clamp, fixator arm, and ankle immobilizer.

Figure 6. Translations of the femoral head in a femur-fixed coordinate frame. 


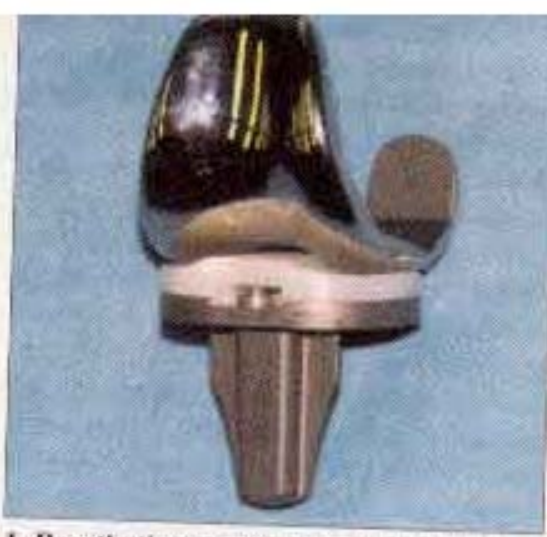

1. Prosthetic components used in total knee replacement surgery.

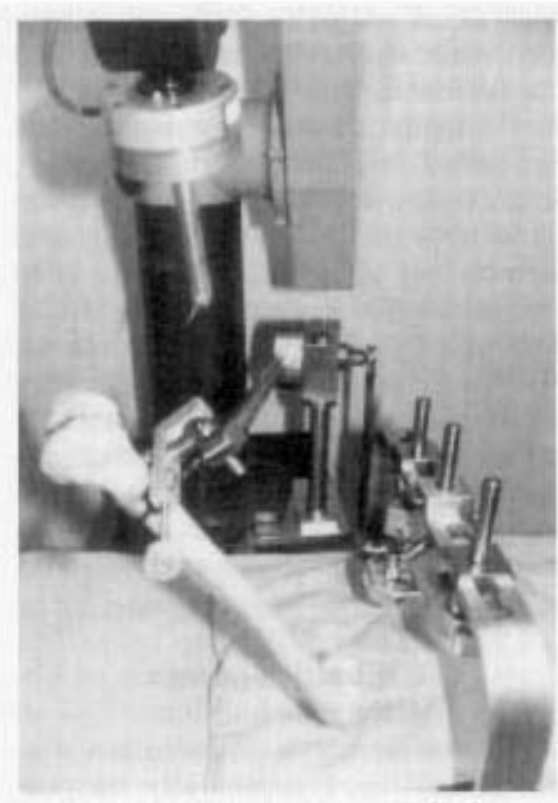

4. Robot, surgical table, and fixturing.

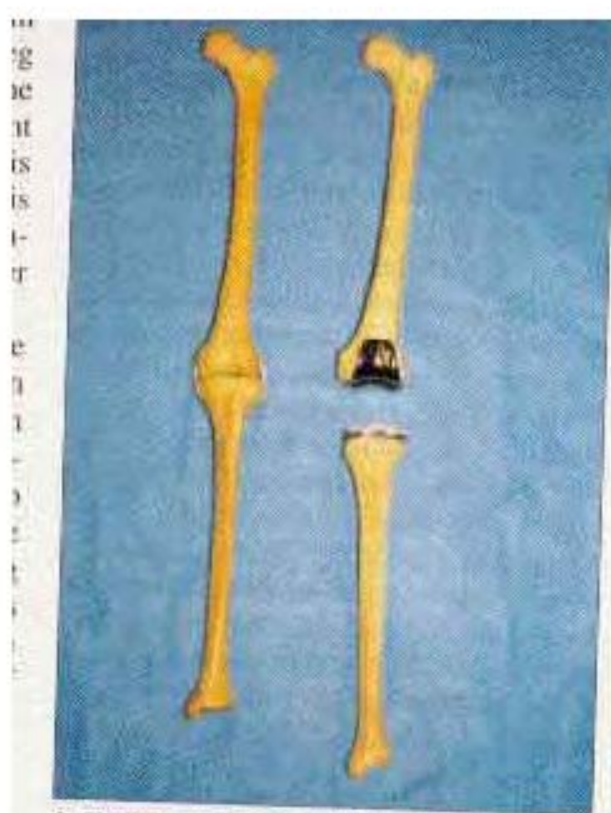

2. Femur and tibia before and after total knee replacement.

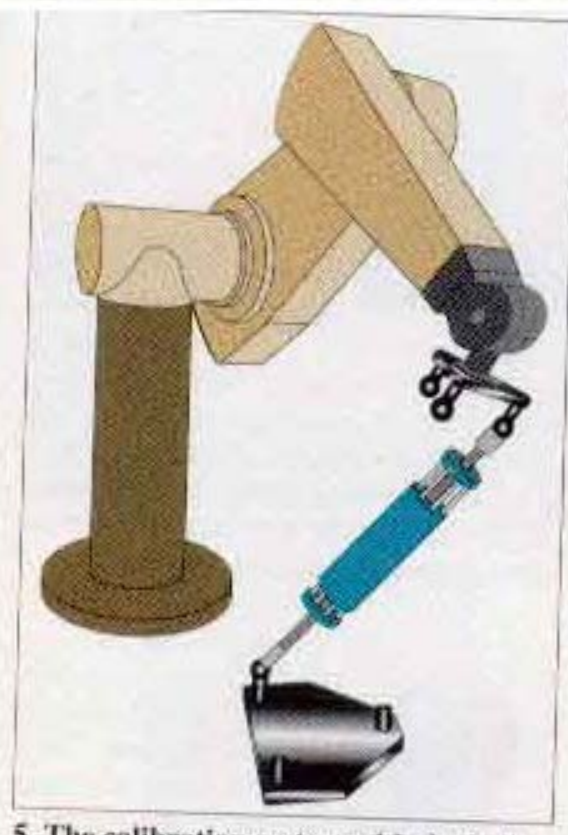

5. The calibration system with the ballbar connected between one of three steel spheres attached to the robot endpoint and a magnetic chuck mounted on the robot tabie.

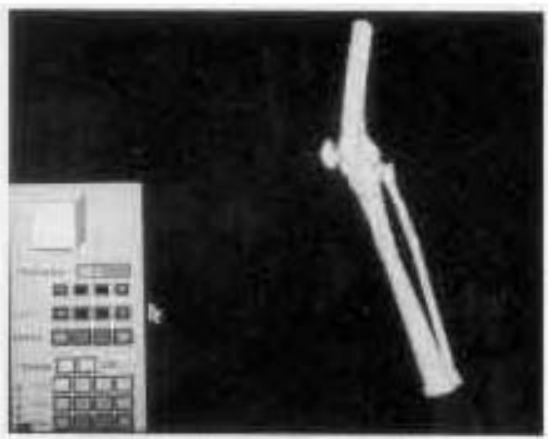

3. 3-D bone reconstruction sereen.

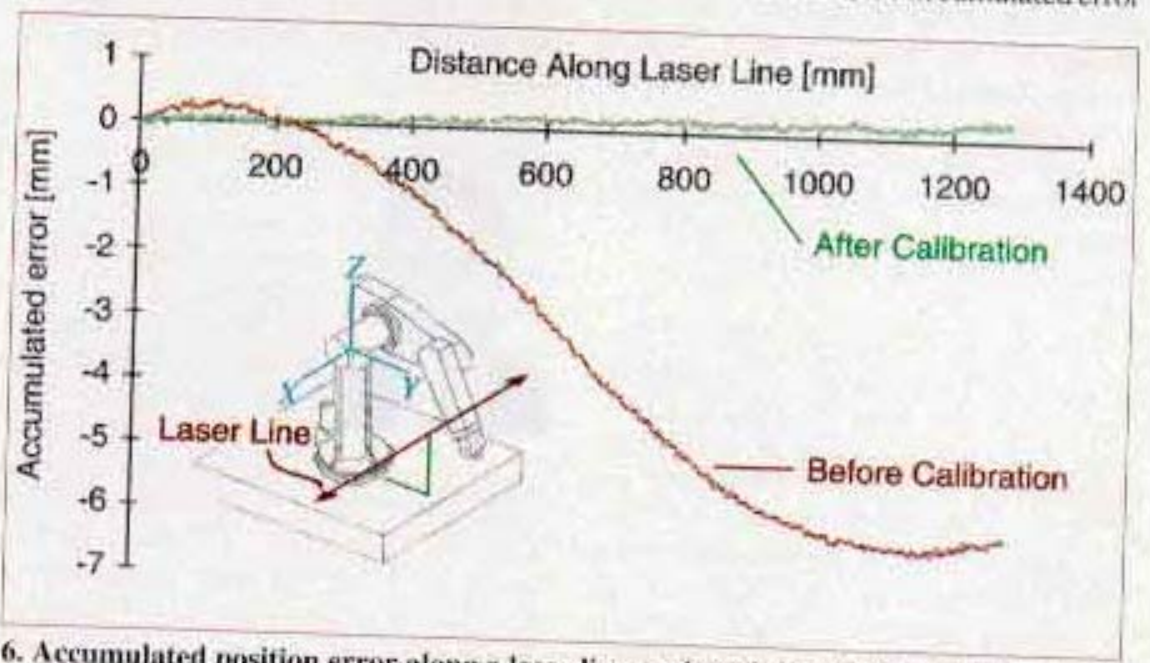

fosition error along a laser line projected through the robot's work space 